$\mathrm{E}$

EVALUAR
2019, Vol. 19, No. 3

ISSN 1667-4545

Recuperado de https://revistas.unc.edu.ar/index.php/revaluar

Laboratorio de Evaluación Psicológica y Educativa

Facultad de Psicología - Universidad Nacional de Córdoba

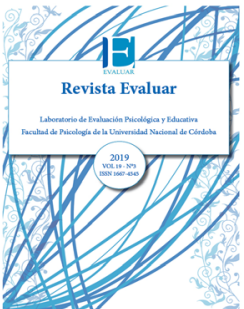

\title{
El factor $p$. ¿La estructura subyacente a la psicopatología?
}

\author{
The $p$ factor. The structure underlying psychopathology?
}

\author{
Silvana Andrea Montes * 1,2, Roberto Oscar Sanchez 2,3 \\ 1 - CONICET (Consejo Nacional de Investigaciones Cientificas y Técnicas), Argentina. \\ 2 - Facultad de Psicología, Universidad Nacional de Mar del Plata, Buenos Aires, Argentina. \\ 3 - Centro de Asistencia Psicológica Mar del Plata (CAPsi), Argentina.
}

Introducción
Método
Resultados
Discusión
Referencias

Recibido: 21/06/2019 Revisado: 20/08/2019 Aceptado: 10/09/2019

\section{Resumen}

La psicopatología se encuentra en un momento de crisis. Los sistemas clasificatorios categoriales han recibido diversas críticas por parte de investigadores y de clínicos. Nuevas propuestas intentan presentar un modelo superador, dimensional y jerárquico. Entre ellas se destacan las que postulan la existencia de un factor general de psicopatología. En este trabajo se buscó analizar la estructura subyacente a los rasgos de personalidad patológicos del modelo dimensional alternativo del DSM-5, buscando aportar evidencia a tales propuestas. Para esto, se examinó la estructura interna de una versión en castellano del Inventario de Personalidad para el DSM-5 [PID-5], mediante análisis factorial confirmatorio. Se compararon cuatro modelos alternativos: el modelo original de cinco factores, de un factor de orden superior, modelo bifactor y unidimensional. El modelo bifactor presentó un mejor ajuste a los datos. Esto sugiere que las variables observadas reflejan cinco rasgos patológicos específicos pero también una propensión general a la psicopatología.

Palabras clave: rasgos patológicos de personalidad, factor general de psicopatología, modelos dimensionales, PID-5, análisis factorial confirmatorio, modelo bifactor

\section{Summary}

Psychopathology is in a moment of crisis. The categorical classification systems have received several criticisms from researchers and clinicians. New proposals try to present a superior, dimensional and hierarchical model. Among them are those that propose a single dimension of general psychopathology. In this study, we sought to analyze the underlying structure of pathological personality traits, looking to provide evidence for such proposals. In doing so, the internal structure of the Spanish version of the Personality Inventory for the DSM-5 [PID-5] was examined by a confirmatory factor analysis. Four alternative models were compared: the five-factor model, a second-ordered factor model, bifactor, and one-factor. The bifactor model presented a better fit to the data. This suggests that the observed variables reflect five specific pathological traits but also a general propensity for psychopathology.

Keywords: personality maladaptive traits, general factor of psychopathology, dimensional models, PID-5, confirmatory factor analysis, bifactor model

*Correspondencia a: Silvana A. Montes, Matheu 212 - 3º B / 7600 - Mar del Plata. Te.: +54 223 4475696. E-mail: smontes@conicet.gov.ar Cómo citar este artículo: Montes, S. A., \& Sanchez, R. O. (2019). El factor p. ¿La estructura subyacente a la psicopatología? Revista Evaluar, 19(3), 20-41. Recuperado de https://revistas.unc.edu.ar/index.php/revaluar 


\section{Introducción}

La psicopatología tradicional, basada en categorías diagnósticas, está en una etapa de crisis. El modelo categorial estuvo presente ya desde la primera versión del Manual Diagnóstico y Estadístico de los Trastornos Mentales (DSM-1; American Psychiatric Association [APA], 1952), si bien alcanzó su mayor esplendor con la publicación de su tercera edición, el DSM-III (APA, 1980). Este fue considerado una verdadera revolución, un cambio de paradigma en el entendimiento de los trastornos mentales (Blashfield, Flanagan, \& Raley, 2010; Coolidge \& Segal, 1998; Mayes \& Horwitz, 2005; Millon, 1983). El acercamiento categorial a la psicopatología implica la existencia de múltiples trastornos individuales, separados, episódicos, distintos y en cantidad creciente. Así, se pasó de 128 trastornos en el DSM-I (APA, 1952) a 347 en el DSM-IV (APA, 1994). Pese a las modificaciones aportadas por las siguientes revisiones del Manual, el modelo no ha resistido el paso del tiempo, y las críticas a su estructura ya son un lugar común (Batstra \& Thoutenhoofd, 2012; Frances, 2010, 2014; Livesley, 2012; Paris, 2012; Verheul, 2012; Widiger, 2013). En un metanálisis realizado con base en 177 artículos, que incluían a más de 530.000 participantes, Haslam, Holland y Kuppens (2012) encontraron que, una vez controlados estadísticamente, solo el 14\% de los hallazgos podían relacionarse con categorías, quedando excluidos los dominios de la personalidad normal, los trastornos del estado de ánimo, los trastornos de ansiedad, los trastornos de la alimentación, los trastornos de externalización y los trastornos de la personalidad (salvo el esquizotípico). La evidencia de categorías psicopatológicas se limitó a la esquizotipia, los trastornos por uso de sustancias y el autismo. Los autores concluyen en que la mayoría de las variables latentes de interés para los psiquiatras, los psicólogos de la personalidad y los clínicos, son dimensionales, y que es probable que muchos hallazgos taxonómicos de influencia en la investigación temprana sean falsos. En los albores de la construcción del DSM-5 (Kupfer, First, \& Regier, 2002) ya se reconocía que el actual sistema quizá nunca pudiera dar cuenta de la etiología subyacente a los trastornos mentales, por lo que resultaría necesario un cambio de paradigma. La alta comorbilidad, los límites arbitrarios entre normalidad y patología, o la pobre cobertura, son ejemplos de las falencias que la comunidad científica viene señalando ya desde hace años (Forbes, Baillie, \& Schniering, 2016; Kessler, Chiu, Demler, \& Walters, 2005; Kupfer et al., 2002; Waszczuk, Kotov, Ruggero, Gamez, \& Watson, 2017), al punto de encontrarse el Manual de la APA (2013) totalmente desacreditado y prácticamente sin uso dentro de la disciplina. La evidencia sugiere que la probabilidad de identificar empíricamente grupos discretos de psicopatología mediante taxonometría no es alta (Krueger et al., 2018), y según Conway et al. (2019) ya existe abundante evidencia de que los enfoques categóricos de los trastornos mentales están obstaculizando el progreso científico. Ha llegado el tiempo de contar con una nueva clasificación de la psicopatología.

La clasificación histórica de los trastornos mentales ha seguido una línea de trabajo que podría considerarse como basada en la autoridad (Krueger et al., 2018). Esto es así ya que en las distintas versiones del DSM han sido grupos de trabajo o paneles de expertos los que han determinado la existencia de las diferentes categorías diagnósticas. Y, si bien luego se buscaba validar empíricamente dichas categorías, esa validación aparecía con posterioridad. Esta metodología puede considerarse como de los conceptos (las categorías) hacia los datos, o de arriba hacia abajo. Por el contrario, las nuevas propuestas intentan ir de los datos hacia los conceptos, o de abajo 
hacia arriba: a partir de resultados empíricos se construye luego el andamiaje teórico que da sentido a esos datos. Sin embargo, no hay que dejar de considerar las advertencias de Muthukrishna y Henrich (2019) cuando sostienen que muchos campos de la Psicología carecen de un marco teórico general que canalice los hallazgos empíricos y que permita derivar predicciones específicas de premisas más generales. La ausencia de tal marco lleva a no saber cuándo esperar que los resultados de una investigación puedan ser replicados y cuándo no. En similar sentido, Caspi y Moffitt (2018) sostienen que, si bien los modelos estadísticos permiten extraer supuestas variables latentes de la relación entre síntomas y trastornos, esto nada nos dice respecto a la estructura teórica subyacente de la psicopatología. Vale decir que sus resultados bien podrían ser un artificio estadístico y no necesariamente representar a una estructura que dé cuenta de la psicopatología. Por tanto, cualquier nueva propuesta, si bien debe partir de datos empíricos concretos, debe poder sostenerse con aportes de otras áreas del conocimiento, en particular genética, neurociencia y psicopatología del desarrollo, que den un marco más amplio a los hallazgos empíricos.

En los últimos años han ido surgiendo nuevas propuestas teóricas, de corte jerárquico y dimensional, y la evidencia parece indicar que tal acercamiento resultaría más apropiado para entender la psicopatología (Lahey et al., 2012; Lahey, Krueger, Rathouz, Waldman, \& Zald, 2017; Caspi et al., 2014; Caspi \& Moffitt, 2018). Dos enfoques alternativos cuentan con evidencia favorable: los modelos de varios niveles (como la Taxonomía Jerárquica de la Patología [HiTOP]; Conway et al., 2019; Krueger et al., 2018; Kotov et al., 2017; Sánchez \& Montes, 2019) y los modelos de un factor general de gravedad y factores específicos que reflejan el estilo (o modelos bifactor; Caspi et al., 2014; Lahey et al., 2012, 2017; Sharp et al.,
2015). Ambas propuestas buscan la mejor manera de conceptualizar la patología. Estadísticamente, ambas resultan adecuadas; las correlaciones entre los factores y las medidas de funcionamiento revelaron asociaciones fácilmente interpretables, lo que respalda la validez de constructo para los dos modelos (Ringwald, Beeney, Pilkonis, \& Wright, 2019). Estas propuestas buscan brindar una estructura más parsimoniosa de la psicopatología que la de los numerosos trastornos categoriales, los cuales no reflejan la estructura natural de los fenómenos psicopatológicos.

Ambos modelos incluyen una dimensión general de psicopatología, denominada "Factor General de Psicopatología" o, sencillamente, "Factor p" (Caspi et al., 2014). Tal denominación alude claramente al factor $g$ de inteligencia general, y así como $g$ refleja distintos grados de inteligencia, de baja a alta, el factor $p$ da cuenta de la severidad de la psicopatología, de baja a alta. Así una única dimensión, $p$, podría medir el riesgo de una mayor propensión a los trastornos mentales, en lo que respecta a la recurrencia de la patología, a la cronicidad, a la comorbilidad y a la gravedad (Caspi et al., 2014). Los modelos, además, están compuestos por una serie de factores de segundo orden (internalización, externalización, trastornos del pensamiento, etc.) que agrupan trastornos con aspectos similares, y que suelen presentar comorbilidad al diagnosticarlos desde la perspectiva tradicional categorial.

En lo que respecta a los trastornos de personalidad (TP), Tyrer (2005) fue el primero en advertir la importancia crucial para la práctica clínica de la gravedad de estos trastornos, aspecto ausente en las distintas versiones del DSM. Cualquier propuesta que pretenda superar al modelo categorial debería incluir una dimensión de gravedad. El autor proponía distinguir la severidad del rasgo del cluster donde el trastorno se expresara. 
Existen diferentes caminos potenciales para alcanzar un modelo psicopatológico basado en la evidencia científica y que sea clínicamente útil. En lo que respecta a los $\mathrm{TP}$, dicha evidencia no permite sostener la idea de que se trate de entidades categóricas ni que se reduzcan a un número discreto de elementos. Sin embargo, como se dijo, la articulación de un modelo superador es un trabajo teórico, además de empírico. Así, los ingentes esfuerzos estadísticos deben ser acompañados por hipótesis teóricas con lazos con otras disciplinas, que refuercen tales hipótesis. Esto es, el logro de una nueva estructura psicopatológica superadora es una tarea que debe ser realizada desde diferentes campos, y no solo desde el acercamiento empírico.

Caspi y Moffitt (2018) revisan la evidencia existente en tal sentido. Por un lado, se cuenta con información que muestra que factores genéticos generales influyen en todas las formas de psicopatología, no solo en lo que respecta a la etiología de trastornos particulares, sino que también podrían explicar la coexistencia de dichos trastornos en la población. Además, diversos estudios han encontrado una tendencia a la activación en ciertas partes del cerebro (por ejemplo amígdala o hipocampo) en personas que cumplían con los criterios para diferentes trastornos psiquiátricos, en comparación con participantes de control sin trastornos (sin embargo, se han registrado pocas diferencias entre los diferentes trastornos). De similar modo, diversos estudios de diferencias volumétricas en personas con diferentes patologías (esquizofrenia, trastorno bipolar, trastorno obsesivo compulsivo, depresión, ansiedad y adicción) encontraron un volumen reducido de materia gris en los diagnósticos en tres regiones cerebrales (corteza cingulada anterior dorsal y corteza insular anterior izquierda y derecha). Para los autores, estos resultados deben estimular futuros esfuerzos para investigar las bases neuronales de la psicopa- tología. Finalmente, la evidencia parece favorecer la hipótesis de la progresión del desarrollo de la psicopatología, donde la comorbilidad secuencial en los mismos individuos sería la norma, al igual que el agravamiento de la patología, respondiendo, en ambos casos, a la hipótesis de un $p$ elevado. Por lo anterior puede sostenerse que, más allá de los aportes de los modelos estadísticos, la evidencia genética, como la neuropsicológica, como la historia psicopatológica individual, son compatibles con la idea de un factor general de psicopatología.

Los trastornos de personalidad son un claro ejemplo de la utilidad de un acercamiento dimensional a la psicopatología, en particular dentro del modelo de los "cinco grandes", donde la idea de un enfoque dimensional de los trastornos de la personalidad viene manejándose desde comienzos de los años 90 (Costa \& Widiger, 1994). En la misma línea, la APA también ha ido avanzando en un modelo dimensional alternativo para los trastornos de la personalidad, tal como aparece en la Sección III (Modelos y medidas emergentes) de la última versión del Manual (APA, 2013). Si bien la propuesta ha recibido duras críticas de diversos sectores de la comunidad científica (Livesley, 2012; Paris, 2012; Verheul, 2012; Sanchez, en prensa; Shedler et al., 2010; Widiger, 2011, 2013), lo cierto es que ha dejado planteada una estructura de cinco dominios para entender la patología de la personalidad: afectividad negativa, desapego, antagonismo, desinhibición y psicoticismo. Fácil es advertir en esas dimensiones los equivalentes patológicos de los "cinco grandes factores de personalidad" (Costa \& Widiger, 1994; John \& Srivastava, 1999; Sanchez \& Ledesma, 2007): neuroticismo, extraversión, amabilidad, responsabilidad y apertura a la experiencia (si bien en este caso la relación con psicoticismo es menos clara). En un trabajo anterior (Sanchez, Montes, \& Somerstein, en prensa) se encontró evidencia 
sobre la estructura dimensional del modelo, al ser analizado con un instrumento desarrollado para su evaluación, en una adaptación para población local. Mediante un análisis factorial exploratorio (AFE) se encontró evidencia de los cinco factores del modelo dimensional de la APA (2013).

\section{La estructura subyacente a la psicopatología}

La alta covariación de los trastornos psicológicos, tanto en un momento dado como a lo largo de la vida, e incluso a través de generaciones, dio lugar a la idea de la existencia de una estructura subyacente a los trastornos mentales. La evidencia inicial para este modelo fue proporcionada por Lahey et al. (2012), y posteriormente Caspi et al. (2014) pudieron replicarla y llamaron "factor $p$ " al supuesto factor de riesgo común para desarrollar todas y cada una de las formas de psicopatología a lo largo del curso de la vida (Caspi \& Moffitt, 2018). Lahey et al. (2012) realizaron análisis factorial confirmatorio (AFC) sobre 11 trastornos prevalentes del DSM-IV en una muestra representativa nacional, y el modelo que mejor se ajustaba a los datos incluía un factor general, que captura lo que esos trastornos tienen en común.

En su trabajo de replicación, Caspi et al. (2014), por su parte, ponen a prueba tres modelos estructurales distintos mediante AFC (los modelos se grafican en la Figura 1). El modelo A, de 3 factores correlacionados, pone a prueba la hipótesis de que existen 3 factores latentes que explican la covariación entre las variables observadas (o los síntomas de los trastornos): internalización, externalización y trastorno del pensamiento. El modelo B retiene un solo factor de orden superior $(p)$, que subsume a los tres factores. Por último, el modelo $\mathrm{C}$, bifactor, agrega un factor que refleja psicopatología general $(p)$. Los resultados del AFC del trabajo de Caspi et al. (2014) sugirieron que el modelo $\mathrm{C}$ fue el que mejor se ajustaba a los datos. Esto significa que internalización, externalización y trastornos del pensamiento agregan información más allá de $p$, de manera que el factor general de psicopatología, por sí solo, no resulta suficiente para describir los datos. De esta forma, el modelo bifactor debe entenderse como la existencia de un factor común como responsable de las distintas formas de psicopatología que, a su vez, coexiste con tres factores independientes que explican un subconjunto más pequeño de síntomas y trastornos.

Ese factor común, entonces, representaría la gravedad de la patología. La gravedad resulta predictora de disfunción social persistente, de la evolución y la cronicidad, de la respuesta al tratamiento y de la calidad de vida en general (Tyrer, 2005). En estudios de criterios o diagnósticos de trastornos de la personalidad, el factor general se ha interpretado como representativo de las características del funcionamiento central de la personalidad, mientras que los factores específicos denotan el estilo de personalidad (vale decir, su contenido); cada elemento proporciona información de diagnóstico única (Hopwood et al., 2011; Ringwald et al., 2019). Esta diferenciación, ausente en las distintas versiones del DSM, explicaría en parte la alta comorbilidad entre TP, ya que los casos más severos cumplirían los criterios para más de un trastorno. El estilo representaría el cómo de la patología, la manera de expresarse la disfunción, mientras que el factor general informaría respecto al cuánto de la patología, al pronóstico del funcionamiento disfuncional. Ambos serían independientes, y los modelos bifactor serían la forma estadística de dar cuenta de su existencia. 


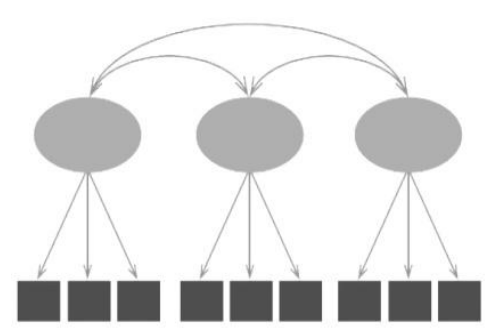

A. Modelo de factores correlacionados

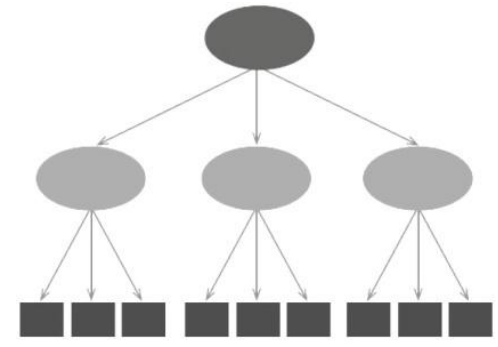

B. Modelo de un factor de orden superior

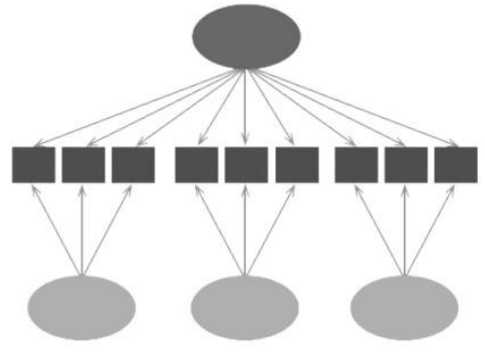

C. Modelo bifactor

\section{Figura 1}

Ilustraciones esquemáticas de las posibles relaciones entre trastornos psicopatológicos (basada en Caspi \& Moffitt, 2018).

\section{Los modelos bifactor}

Los modelos bifactor, de reciente aplicación en psicopatología, hunden sus raíces históricas en la investigación pionera de la inteligencia (Holzinger \& Swineford, 1937). En líneas generales, se los considera los más adecuados para evaluar simultáneamente la influencia de factores generales y específicos tras un conjunto de ítems (Dominguez-Lara \& Rodriguez, 2017). Reciben cada vez más atención en su aplicación a medidas de personalidad y de psicopatología, buscando demostrar la existencia de un factor general de psicopatología (ver por ej., Caspi et al., 2014; Lahey et al., 2012). En este modelo, cada variable posee cargas en dos factores: un factor general en donde cargan todas las variables (en este caso $p$ ), y un factor específico, que influye solo en un conjunto de variables observadas (por ejemplo, ansiedad cargaría en su factor, afectividad negativa, pero también en el factor general).

A diferencia del modelo de segundo orden (por ej., Modelo B en la Figura 1), el factor general tiene un efecto directo sobre los indicadores o variables observados (ítems; Hoyle, 2012) e influye en todos los ítems de forma simultánea a los factores específicos. Además, en un modelo bifactor, los factores específicos suelen ser modelados ortogonalmente ya que se parte de la hipótesis de que la varianza compartida entre ellos se debe a la presencia de un factor general (Reise, 2012; Rodriguez, Reise, \& Haviland, 2016). Estos modelos son especialmente útiles para evaluar la validez de un instrumento destinado a medir tanto el concepto global como sus dimensiones específicas (Rodriguez et al., 2016). No obstante, no están exentos de problemas o controversias, especialmente si se aplican al campo de la psicopatología (Bonifay, Lane, \& Reise, 2017). Al respecto, muchos instrumentos que se consideran bifactor son esencialmente unidimensionales y la interpretación de los factores específicos termina siendo forzada (ver por ej., Arias, Ponce, \& Nuñez, 2018; Reise, Kim, Mansolf, \& Widaman, 2016). Tal como señala Reise et al. (2016), se debe ser cauteloso al concluir en que el modelo bifactor es el mejor solo sobre la base de que es superior el ajuste. Por esta razón, varios autores recomiendan utilizar diferentes criterios a la hora de evaluar el ajuste de modelos bifactor. Dichos criterios deben incluir: a) consideraciones teóricas y b) indicadores específicos del modelo bifactor (Arias et al., 2018; Domiguez-Lara \& Rodriguez, 2017; Flores-Kanter, Dominguez-Lara, Trólogo, \& Medrano, 2018; Rodriguez et al., 2016). No obstante, a pesar de estar recomendados, su uso se encuen- 
tra aún poco extendido entre los investigadores en Psicología. De hecho, los estudios que sugieren la presencia de un factor general de psicopatología (Caspi et al., 2014; Lahey et al., 2012; Snyder, Young, \& Hankin, 2017) no han reportado tales indicadores -p. ej., el ECV (explained common variance; Sijtsma, 2009; Ten-Berge \& Socăn, 2004) o el $\omega$ h (coeficiente omega jerárquico; Zinbarg, Yovel, Revelle, \& McDonald, 2006).

\section{Justificación y objetivo}

Tradicionalmente, los modelos más utilizados para analizar de manera dimensional datos sobre personalidad o sobre trastornos de la personalidad han sido el de cinco factores correlacionados y modelos jerárquicos que postulan una dimensión de orden superior por sobre los factores (Gutiérrez et al., 2017; Watters \& Bagby, 2018). En la literatura, no abundan trabajos realizados a partir de propuestas diferentes. Por otro lado, en los últimos años, han proliferado las aplicaciones de los modelos bifactor en el ámbito de evaluación clínica y la psicopatología (Caspi et al., 2014, Lahey et al., 2012; Patrick, Hicks, Nichol, \& Krueger, 2007; Urbán, Arrindell, Demetrovics, Unoka, \& Timman, 2016). Al mismo tiempo, la estructura pentafactorial del PID-5 (Krueger, Derringer, Markon, Watson, \& Skodol, 2012) ha sido replicada en muchos estudios psicométricos (ver por ej., Al-Dajani, Gralnick, \& Bagby, 2016; Watters \& Bagby, 2018); no obstante, según el conocimiento de los autores, aún no se han aplicado modelos bifactor a los rasgos patológicos de personalidad propuestos por el modelo alternativo del DSM-5 o para explicar la dimensionalidad de los puntajes del PID-5. Es posible pensar, en línea con Caspi et al. (2014) y Caspi y Moffitt (2018), la existencia de un factor general de psicopatología $(p)$, además de los cinco factores del modelo original.
En consecuencia, el objetivo de este trabajo es analizar la estructura subyacente a los rasgos de personalidad patológicos del modelo dimensional alternativo del DSM-5 (APA, 2013). Para ello, se examinó la estructura interna de una versión en castellano del Inventario de Personalidad para el DSM-5 [PID-5] (Sanchez et al., en prensa). Los diferentes modelos estructurales de la psicopatología fueron sometidos a prueba a través de AFC. Específicamente, se compararon cuatro modelos alternativos: el modelo original de cinco factores correlacionados, un modelo de un factor de orden superior, un modelo bifactor y el modelo unidimensional. Estos se describen con mayor detalle a continuación.

\section{Modelos testeados}

Se probaron varias hipótesis sobre la estructura interna de la versión al castellano del PID-5 (Sanchez et al., en prensa): Modelo 1 (M1) con cinco factores oblicuos (modelo dimensional alternativo del DSM-5); Modelo 2 (M2) con cinco factores de primer orden y un factor de segundo orden ( $p$ ); Modelo 3 (M3), bifactor, con un factor general $(p)$ y cinco factores específicos; y Modelo 4 (M4), unidimensional (con un único factor general $p$ ).

El M1 es el que corresponde a la estructura factorial original y es el más utilizado en investigaciones previas (A1-Dajani et al., 2016; Krueger et al., 2012; Sanchez et al., en prensa). Con este modelo, se testea la hipótesis de que hay cinco factores latentes que reúnen rasgos comunes: desapego, afectividad negativa, desinhibición, psicoticismo y antagonismo. El modelo asume que estos factores están correlacionados. Dadas las correlaciones entre estos rasgos latentes, cabe pensar en un factor de orden superior (segundo orden) en el cual convergen los cinco factores de 
primer orden. Es decir, el factor general de psicopatología (Caspi et al., 2014; Caspi \& Moffitt, 2018). Esto se testea en el modelo M2. El tercer modelo, bifactor (M3), es otra forma de representar a $p$. Este modelo se caracteriza tanto por una dimensión única (un factor general, en adelante FG), así como también por un conjunto de factores específicos que influyen en las variables observadas (en adelante, FEs). Por último, en el M4 se testea un modelo unidimensional, con un único factor general $p$ que surge como factor latente desde las variables observadas (i.e., todos los ítems son influidos por una sola variable latente).

\section{Método}

Participantes

Se reanalizaron datos del estudio original de validación del PID-5 en Argentina (Sanchez et al., en prensa). La muestra (no probabilística) consistió en 393 participantes de población general. Sus edades estaban comprendidas entre los 18 y 85 años $($ Media $=39.3 ; \mathrm{DE}=15.5)$; el $68.4 \%$ eran mujeres (269) y el $31.6 \%$ hombres (124). La mayoría de los participantes, el 54.2\%, tenía nivel universitario de educación (213), el 18.1\% nivel terciario (71), el 13.7\% secundario (54), el 13.5\% posgrado (53) y el $0.5 \%$ educación primaria, en todos los casos, completa o incompleta.

\section{Instrumentos}

Inventario de Personalidad para el DSM-5 (PID-5; Krueger et al., 2012). El PID-5 evalúa los cinco dominios de personalidad patológica del modelo dimensional alternativo del DSM-5. Se solicita a la persona que responda de la forma más honesta posible y que seleccione la opción de respuesta que mejor lo describe como es general- mente. En el trabajo anterior (Sanchez et al., en prensa) se detallan las consideraciones seguidas en la construcción de la versión local. Cabe señalar que el instrumento original cuenta con dos versiones, la extensa (220 ítems) y la breve (25 ítems). En esta primera versión local se utilizaron los 25 ítems de la versión breve, algunos de ellos con cambios menores para adecuarlos a nuestro contexto. Para tratar de contemplar la mayor cantidad de facetas del instrumento y la posible eliminación de ítems por un funcionamiento psicométrico defectuoso, se agregaron 13 ítems, lo que dio lugar a una versión preliminar de 38 (10 tomados de la versión extensa del instrumento y 3 creados con la intención de reflejar las facetas faltantes). De este modo, en esta versión preliminar quedaron incluidas 24 de las 25 facetas del modelo (restando perfeccionismo rígido, que puntúa en desinhibición cuando está ausente). Finalmente, 7 de los ítems mostraron una carga menor a .30, por lo que fueron descartados, y se optó por excluirlos de los análisis posteriores. De esta forma, la versión final en castellano constaba de 31 ítems.

\section{Procedimiento}

Se contactó a los participantes por correo electrónico, mediante un procedimiento no probabilístico (muestreo de bola de nieve). Fueron reclutados en un período de 60 días (noviembre y diciembre de 2016). Los datos fueron recolectados a través de una página web diseñada para este trabajo. La toma de datos se realiza de manera autoadministrada y la plataforma va guiando al participante al responder los reactivos. Antes del protocolo de respuesta se agregó un consentimiento informado, donde se señalaban las características y el propósito de la investigación, y se garantizaba el anonimato en la administración de los instrumentos y en el tratamiento de los datos. 
La aceptación de este consentimiento resultaba obligatoria para pasar a la página de toma de datos. Por el propio diseño de la plataforma, no se registraron datos faltantes.

\section{Análisis de datos}

En primer término, para conocer cómo se distribuyen los datos y evaluar el método de estimación más adecuado, se calculó para cada ítem la media, la desviación estándar, la asimetría y la curtosis (ver Tabla 1). Además, se realizó una prueba de normalidad univariada a través del coeficiente de Kolmogorov-Smirnov con la corrección Lilliefors, y la prueba de normalidad multivariada de Mardia (1970).

Posteriormente, se aplicó un AFC para testear los diferentes modelos. El ajuste de los modelos fue interpretado a partir de los siguientes índices: a) Absolutos: $\chi^{2}$ (debe ser lo más bajo posible y no significativo — adecuado $p>.05-)$; Satorra-Bentler $\chi^{2}$ (S-B; Satorra \& Bentler, 1990), un indicador más robusto que el $\chi^{2}$, ya que tiene en cuenta la desviación de la multinormalidad en las variables; GFI (goodness-of-fit index; adecuado $p>$.90); AGFI (adjusted goodness-of-fit index; adecuado $p>.90$ ); RMSEA (root-mean-square error of approximation; adecuado $p<.08$ ); SRMR (standardized root mean square residual; adecuado $p<.08$ ); b) Comparativos: CFI (comparative fit index; adecuado $p>90$ ); IFI (incremental fit index; adecuado $p>-90$ ), NFI (normed fit index; adecuado $p>.90$ ); NNFI (non normed fit index; adecuado $p>.90$ ); y c) De parsimonia (también llamados information criteria): AIC Modelo (Akaike information criterion); CAIC Modelo (consistent Akaike information criterion). Estas medidas se utilizan sobre todo para la comparación de modelos alternativos. En ambos casos, valores altos indican peor ajuste (Van de Schoot,
Lugtig, \& Hox, 2012). Se calculó la diferencia entre el CAIC Independiente y el CAIC Modelo (CI-CM), donde mayores diferencias indican mejor ajuste. Todos los ítems fueron modelados en el factor teóricamente esperado y de acuerdo con los resultados del AFE de Sanchez et al. (en prensa). Se utilizaron el software LISREL 8.80 for Windows (Jöreskog \& Sörbom, 2006) para el AFC y el programa SPSS y Factor (Lorenzo-Seva \& Ferrando, 2013) para el resto de los análisis.

En el modelo bifactor, además, se calcularon indicadores específicos siguiendo las recomendaciones de la literatura previa (Arias et al., 2018; Flores-Kanter et al., 2018; Rodriguez et al., 2016), con el módulo propuesto por DominguezLara y Rodriguez (2017). Estos fueron el ECV (explained common variance; Sijtsma, 2009; Ten-Berge \& Socăn, 2004), el ECV-I (Stucky, Thissen, \& Edelen, 2013), el PUC (percentage of uncontaminated correlations), el omega jerárquico ( $\omega \mathrm{h}$; Zinbarg et al., 2006), el omega jerárquico subescala ( $\omega \mathrm{hs}$; Reise, 2012) y el coeficiente H (Hancock, 2001; Gagne \& Hancock, 2006). El ECV es el porcentaje de varianza común explicada por el factor general y es un indicador de unidimensionalidad (Rodriguez et al., 2016). El ECV-I es la aplicación del ECV a nivel del ítem (Stucky et al., 2013) e indica qué porcentaje de la varianza verdadera de cada ítem es explicada por el FG (se esperan valores $\geq .80$ para concluir una influencia significativa del FG sobre los ítems; Stucky \& Edelen, 2014; como se citó en Rodriguez et al., 2016).

El PUC (porcentaje de correlaciones no contaminadas por la multidimensionalidad) se utiliza conjuntamente con el ECV para decidir si los datos son esencialmente unidimensionales. Un ECV por encima de .90 sugiere que los datos son completamente unidimensionales, mientras que un ECV por debajo de .70 indica que los datos son multidimensionales (Quinn, 2014). En 
este caso, por razones teóricas (modelo de cinco factores previamente mencionado) se espera un ECV bajo o moderado.

El omega jerárquico $(\omega \mathrm{h})$ permite evaluar la fortaleza del FG y también puede calcularse para cada FE ( $\omega \mathrm{hs})$. El $\omega$ h es la proporción de la varianza explicada por el factor general y las whs la proporción de la varianza explicada por cada factor específico, cuando se controla la varianza debida al factor general (Smits, Timmerman, Barelds, \& Meijer, 2014). En cuanto al $\omega$ h se esperan magnitudes $\geq .70$ para concluir, al menos parcialmente, a favor de la unidimensionalidad y para el caso del $\omega$ hs valores $\geq .30$ podrían considerarse como significativos (Dominguez-Lara \& Rodriguez, 2017; Smits et al., 2014). En cuanto al coeficiente $\mathrm{H}$, se puede tomar como una medida de confiabilidad del constructo más robusta que los coeficientes $\alpha$ y $\omega$. Se trata de una estimación de estabilidad y replicabilidad de constructo para cada factor. Un $\mathrm{H} \geq .70$ indica menor probabilidad de fluctuaciones de las cargas factoriales entre varias muestras (Hancock, 2001). Altos valores de $\mathrm{H}$ sugieren mayor estabilidad tanto del factor como de las relaciones con otras variables. Si uno de los factores específicos obtiene un coeficiente $\mathrm{H} \geq .70$, puede considerarse como un factor de grupo robusto, además del factor general (Dominguez-Lara, 2016).

\section{Resultados}

Análisis preliminar de los items

Como se muestra en la Tabla 1, se observaron distribuciones asimétricas tanto positivas como negativas con valores diversos de curtosis, lo que sugiere la presencia de no-normalidad multivariada. La prueba de Kolmogorov-Smirnov con un nivel de significación de $p<.001$ para todos los ítems sugiere rechazar la hipótesis nula de que las variables se distribuyen conforme a la distribución normal. El coeficiente de Mardia (1970) presentó índices de asimetría y curtosis significativas $(p<.001)$, lo que permite rechazar la hipótesis nula de distribución normal multivariante. En consecuencia, se utilizó el método de estimación DWLS (robust diagonally weighted least squares), un método robusto frente a la violación del supuesto de normalidad (requiere muestras amplias n > 200; Li, 2016; Mîndrilă, 2010).

\section{Tabla 1}

Media, desviación estándar, asimetría, curtosis y prueba de Kolmorogov-Smirnof para los ítems del PID-5.

\begin{tabular}{ccccccc}
\hline Ítem & Media & DE & $\begin{array}{c}\text { Asime- } \\
\text { tría }\end{array}$ & $\begin{array}{c}\text { Curto- } \\
\text { sis }\end{array}$ & K-S & p \\
\hline 1 & 1.60 & 0.93 & 1.55 & 1.80 & .37 & $<.001$ \\
2 & 3.53 & 1.23 & -0.48 & -0.80 & .22 & $<.001$ \\
3 & 2.10 & 1.21 & 0.78 & -0.54 & .26 & $<.001$ \\
4 & 2.44 & 1.28 & 0.44 & -0.93 & .19 & $<.001$ \\
5 & 2.01 & 1.19 & 0.87 & -0.37 & .29 & $<.001$ \\
6 & 2.14 & 1.24 & 0.76 & -0.58 & .26 & $<.001$ \\
7 & 1.93 & 1.13 & 1.05 & 0.17 & .29 & $<.001$ \\
8 & 2.18 & 1.14 & 0.59 & -0.64 & .22 & $<.001$ \\
9 & 1.62 & 1.01 & 1.61 & 1.64 & .39 & $<.001$ \\
10 & 2.08 & 1.18 & 0.88 & -0.17 & .25 & $<.001$ \\
11 & 1.81 & 1.17 & 1.35 & 0.73 & .34 & $<.001$ \\
12 & 3.51 & 1.31 & -0.49 & -0.90 & .19 & $<.001$ \\
13 & 2.09 & 1.34 & 0.96 & -0.34 & .29 & $<.001$ \\
14 & 3.26 & 1.36 & -0.26 & -1.10 & .16 & $<.001$ \\
15 & 1.91 & 0.99 & 1.20 & 1.36 & .24 & $<.001$ \\
16 & 1.48 & 0.98 & 2.20 & 4.06 & .43 & $<.001$ \\
17 & 2.12 & 1.33 & 0.90 & -0.46 & .28 & $<.001$ \\
18 & 2.12 & 1.24 & 0.85 & -0.35 & .25 & $<.001$ \\
19 & 1.77 & 1.09 & 1.38 & 1.02 & .33 & $<.001$ \\
21 & 2.39 & 1.43 & 0.55 & -1.09 & .24 & $<.001$ \\
22 & 2.31 & 1.25 & 0.66 & -0.51 & .20 & $<.001$ \\
23 & 2.07 & 1.21 & 0.82 & -0.45 & .28 & $<.001$ \\
24 & 1.46 & 0.92 & 2.15 & 4.00 & .44 & $<.001$ \\
25 & 2.51 & 1.33 & 0.32 & -1.13 & .20 & $<.001$ \\
26 & 2.14 & 1.20 & 0.78 & -0.35 & .24 & $<.001$ \\
27 & 1.84 & 1.11 & 1.34 & 1.04 & .29 & $<.001$ \\
28 & 3.74 & 1.25 & -0.80 & -0.28 & .22 & $<.001$ \\
29 & 2.08 & 1.20 & 0.87 & -0.31 & .25 & $<.001$ \\
30 & 1.69 & 1.12 & 1.61 & 1.62 & .37 & $<.001$ \\
31 & 2.24 & 1.28 & 0.69 & -0.64 & .23 & $<.001$ \\
\hline & & & & & &
\end{tabular}

Nota. ${ }^{1}$ Corrección de la significación de Lilliefors. 
Tabla 2

Índices de ajuste para los modelos testeados.

\begin{tabular}{|c|c|c|c|c|c|c|c|c|c|c|c|c|c|c|}
\hline Modelo & $\chi^{2}$ & $d f$ & $\begin{array}{l}\text { Satorra- } \\
\text { Bentler } \chi^{2}\end{array}$ & CFI & $\begin{array}{c}\text { RMSEA } \\
\text { [ 90\% IC] }\end{array}$ & SRMR & GFI & AGFI & IFI & NFI & NNFI & $\begin{array}{c}\text { AIC } \\
\text { MODEL }\end{array}$ & $\begin{array}{c}\text { CAIC } \\
\text { MODEL }\end{array}$ & CI-CM² \\
\hline $\begin{array}{l}\text { M1. Cinco } \\
\text { factores } \\
\text { oblicuos }\end{array}$ & 2211.24 & 395 & 1079.36 & .940 & $\begin{array}{c}.065 \\
{[.060 ; .070]}\end{array}$ & .087 & .936 & .925 & .940 & .908 & .934 & 1200.87 & 1549.04 & 10138.44 \\
\hline $\begin{array}{l}\text { M2. Cinco } \\
\text { factores } \\
\text { y uno de } \\
\text { segundo } \\
\text { orden (p) }\end{array}$ & 2239.04 & 400 & 1060.87 & .938 & $\begin{array}{c}.065 \\
{[.061 ; .070]}\end{array}$ & .089 & .931 & .920 & .939 & .906 & .933 & 1209.36 & 1532.66 & 10154.82 \\
\hline $\begin{array}{l}\text { M3. } \\
\text { Bifactor }\end{array}$ & 1913.05 & 375 & 905.95 & .950 & $\begin{array}{c}.060 \\
{[.055 ; .065]}\end{array}$ & .082 & .940 & .930 & .950 & .920 & .940 & 1085.95 & 1533.59 & 10.153 .89 \\
\hline $\begin{array}{l}\text { M4. Unidi- } \\
\text { mensional }\end{array}$ & 4039.20 & 405 & 2061.79 & .850 & $\begin{array}{c}.102 \\
{[.098 ; .107]}\end{array}$ & .114 & .876 & .857 & .850 & .820 & .839 & 2181.79 & 2480.21 & 9207.27 \\
\hline
\end{tabular}

Nota. La probabilidad asociada a todos los $\chi^{2}$ de la tabla es $p<0.001$. CAIC Independence - CAIC Model. Valor de CAIC Independence (11687.48).

\section{Análisis factorial confirmatorio}

En primer lugar, se eliminó el ítem 20 dada su baja carga factorial en todos los modelos testeados. Se trata de un ítem inverso (Habitualmente muestro mis emociones a los demás) y que en el AFE original (Sanchez et al., en prensa) presentaba cargas en dos factores: en su original, desapego (.50), y en afectividad negativa (-.31) y un bajo índice de discriminación (correlación ítemtest .29). Luego de eliminarlo, todos los modelos mostraron mejores índices de ajuste. En la Tabla 2 se muestran valores detallados de cada uno de los índices. Tal como se observa en la tabla, a excepción del Modelo 4 (unidimensional), todos los modelos obtuvieron un buen ajuste ya que alcanzaron valores apropiados de CFI $(>.90)$ y RMSEA ( < .08). No obstante, el modelo bifactor fue el que globalmente tuvo mejor ajuste: CFI ( $>$ $.95)$; RMSEA $(<.06)$, y fue el único con valores de SRMR adecuados $(<.08$; Browne \& Cudeck, 1992; Hu \& Bentler, 1999).

El primer modelo, de cinco factores correlacionados, se ajustó bien a los datos: $\chi^{2}=$
$2211.24_{3955}, p<.001 ; \mathrm{S}-\mathrm{B} \chi^{2}=1079.36 ; \mathrm{CFI}=$ $.940 ;$ RMSEA $=.065,90 \%$ IC $=[.060 ; .070]$; GFI $=.936 ; \mathrm{AGFI}=.925 ; \mathrm{IFI}=.94 ; \mathrm{NFI}=.908 ; \mathrm{NNFI}$ $=.934$. Los parámetros estimados fueron todos positivos y oscilaron entre .30 y .91 . Las correlaciones entre los factores fueron todas positivas y oscilaron entre .18 (p. ej., entre antagonismo y desapego) y .68 (p. ej., entre afectividad negati$v a$ y desinhibición, y entre afectividad negativa y psicoticismo; ver Tabla 3). Estas correlaciones son coherentes con las reportadas en el artículo de Sanchez et al. (en prensa) aunque en general tienden a ser más elevadas (p. ej., en el trabajo citado las correlaciones entre afectividad negativa y desinhibición eran de .46 y entre afectividad negativa y psicoticismo .52).

El Modelo 2, con un factor de segundo orden, también tuvo un buen ajuste, aunque levemente inferior al Modelo 1: $\chi^{2}=2239.04{ }_{(400)}, p$ $<.001 ; \mathrm{S}-\mathrm{B} \chi^{2}=1060.87$; CFI $=.938$; RMSEA $=$ $.065,90 \% \mathrm{IC}=[.061 ; .070] ; \mathrm{GFI}=.931 ;$ AGFI $=.920 ; \mathrm{IFI}=.939 ; \mathrm{NFI}=.906 ; \mathrm{NNFI}=.933$. No obstante, este modelo es el que presenta mejores valores de CAIC (1532.66) y CI-CM (10154.82), 
Tabla 3

Cargas factoriales y correlaciones entre los factores en el Modelo 1 de cinco factores correlacionados.

\begin{tabular}{|c|c|c|c|c|c|c|}
\hline & Item & Desapego & $\begin{array}{c}\text { Afecti- } \\
\text { vidad } \\
\text { negativa }\end{array}$ & $\begin{array}{l}\text { Psicoticis- } \\
\text { mo }\end{array}$ & $\begin{array}{l}\text { Antago- } \\
\text { nismo }\end{array}$ & $\begin{array}{l}\text { Desinhi- } \\
\text { bición }\end{array}$ \\
\hline 1 & La gente me describiría como imprudente. & & & & & .61 \\
\hline 2 & Me preocupo por casi todo. & & .39 & & & \\
\hline 3 & Siento que nada de lo que hago es importante. & .79 & & & & \\
\hline 4 & A veces, mis pensamientos o ideas no tienen sentido para los demás. & & & .78 & & \\
\hline 5 & No estoy muy interesado en hacer amigos. & .68 & & & & \\
\hline 6 & Me gusta llamar la atención. & & & & .59 & \\
\hline 7 & Casi nunca me entusiasmo mucho con algo. & .64 & & & & \\
\hline 8 & Suelo hacer lo que los demás quieren que haga. & & .50 & & & \\
\hline 9 & Tengo facilidad para aprovecharme de los demás. & & & & .91 & \\
\hline 10 & Siento que actúo totalmente por impulso. & & & & & .66 \\
\hline 11 & $\begin{array}{l}\text { Con frecuencia, me quedo "en blanco" y de repente me doy cuenta de } \\
\text { que ha pasado mucho tiempo. }\end{array}$ & & & .67 & & \\
\hline 12 & Me emociono fácilmente, incluso por pequeñas cosas. & & .30 & & & \\
\hline 13 & Evito las relaciones románticas. & .47 & & & & \\
\hline 14 & Soy una persona muy ansiosa. & & .54 & & & \\
\hline 15 & Los demás me ven como una persona muy responsable. & & & & & .29 \\
\hline 16 & A veces he visto cosas que en realidad no estaban ahí. & & & .67 & & \\
\hline 17 & Temo estar solo en la vida más que a cualquier otra cosa. & & .51 & & & \\
\hline 18 & $\begin{array}{l}\text { Me empecino en hacer las cosas de una manera, aun cuando es claro } \\
\text { que no va a funcionar. }\end{array}$ & & .68 & & & \\
\hline 19 & Puedo utilizar a la gente para conseguir lo que quiero. & & & & .87 & \\
\hline 21 & $\begin{array}{l}\text { Tengo pensamientos que tienen sentido para mí, pero resultan extraños } \\
\text { para otros. }\end{array}$ & & & .75 & & \\
\hline 22 & Me enojo fácilmente por todo tipo de cosas. & & .60 & & & \\
\hline 23 & No me gusta estar demasiado cerca de la gente. & .70 & & & & \\
\hline 24 & Las cosas que están a mi alrededor a veces me parecen irreales. & & & .69 & & \\
\hline 25 & Creo que otros se aprovecharían de mí si pudieran. & & .68 & & & \\
\hline 26 & $\begin{array}{l}\text { Aun sabiendo lo que es mejor, no puedo dejar de tomar decisiones } \\
\text { precipitadas. }\end{array}$ & & & & & .74 \\
\hline 27 & Disfruto estar enamorado. & .34 & & & & \\
\hline 28 & Puedo ser encantador cuando quiero lograr algo. & & & & .41 & \\
\hline 29 & A veces hago promesas que sé que no voy a poder cumplir. & & & & & .61 \\
\hline 30 & En ocasiones no llego a diferenciar si algo lo viví, lo soñé o lo imaginé. & & & .60 & & \\
\hline 31 & $\begin{array}{l}\text { Me gusta hacer las cosas rápido, aunque queden errores o detalles por } \\
\text { resolver. }\end{array}$ & & & & & .46 \\
\hline
\end{tabular}

\section{Desapego}

Afectividad Negativa

Psicoticismo

Antagonismo

Desinhibición
.57

.61

.18

.38
.68

.39

.68
.36

$.63 \quad .52$

Nota. *Se eliminó ítem 20 de la versión de Sanchez et al. (en prensa). 
pero se aleja de valores adecuados de SRMR $(p<$ .089). Como se ha mencionado, cuando se comparan modelos, valores menores AIC y CAIC indican mejor ajuste. Las cargas factoriales oscilaron entre $.33 \mathrm{y} .87$, y resultan muy similares al Modelo 1, aunque en algunos ítems mejoran levemente. Las correlaciones entre el factor de segundo orden $(p)$ y los factores de primer orden fluctuaron entre .48 y .85 . También se observaron correlaciones entre los factores de primer orden (entre .30 y .68; ver Tabla 4). Estas también son bastante similares a las del Modelo 1.

El tercer modelo bifactor (ver Figura 2) es el que presenta mejor ajuste a los datos: $\chi^{2}=$ $1913.05_{(375)}, p<.001 ; \mathrm{S}-\mathrm{B} \chi^{2}=905.95 ; \mathrm{CFI}=.95$; $\mathrm{RMSEA}=.060,90 \% \mathrm{IC}=[.055 ; .065] ; \mathrm{GFI}=$ $.940 ; \mathrm{AGFI}=.930 ; \mathrm{IFI}=.950 ; \mathrm{NFI}=.920$; NNFI $=.940 . \mathrm{S}-\mathrm{B} \chi^{2}$, resultó el más bajo de todos los modelos. Nótese que es el único que alcanza un CFI de .95. Si bien algunos investigadores sugieren que el punto de corte del CFI es $>.90$, otros más exigentes sugieren que debe ser $>.95(\mathrm{Hu}$ \& Bentler, 1999; Van de Schoot et al., 2012). Además, sólo este modelo se acerca al punto de corte adecuado de SRMR $(<.08)$ y presenta valores óptimos de RMSEA ( $p<.06$; Hu \& Bentler, 1999). En cuanto a AGFI, IFI, NFI y NNFI, estos poseen los valores más elevados. No obstante, se debe notar que el CAIC (1533.59) y el CICM (10153.89) fueron levemente mejores en el Modelo 2, aunque las diferencias son mínimas.

Como se puede ver en la Tabla 5, las magnitudes de las cargas factoriales de los FEs difieren de las cargas factoriales de los FG en el factor correspondiente. La contribución de los FEs en algunos casos está por encima del FG, y en otros casos es al revés. Si bien la mayoría de los ítems tienen cargas por encima de .30, hay algunas excepciones de ítems que poseen cargas muy bajas en el FE, pero más elevadas en el FG y viceversa (como es el caso del ítem 25, que en el
Tabla 4

Cargas factoriales y correlaciones entre los factores en el Modelo 2, de segundo orden.

\begin{tabular}{|c|c|c|c|c|c|}
\hline & Desapego & $\begin{array}{c}\text { Afectividad } \\
\text { negativa }\end{array}$ & $\begin{array}{c}\text { Psico- } \\
\text { ticismo }\end{array}$ & $\begin{array}{c}\text { Antago- } \\
\text { nismo }\end{array}$ & $\begin{array}{c}\begin{array}{c}\text { Desinhibi- } \\
\text { ción }\end{array} \\
\end{array}$ \\
\hline 1 & & & & & .61 \\
\hline 2 & & .39 & & & \\
\hline 3 & .79 & & & & \\
\hline 4 & & & .78 & & \\
\hline 5 & .69 & & & & \\
\hline 6 & & & & .57 & \\
\hline 7 & .66 & & & & \\
\hline 8 & & .50 & & & \\
\hline 9 & & & & .93 & \\
\hline 10 & & & & & .66 \\
\hline 11 & & & .67 & & \\
\hline 12 & & .30 & & & \\
\hline 13 & .47 & & & & \\
\hline 14 & & .54 & & & \\
\hline 15 & & & & & .31 \\
\hline 16 & & & .67 & & \\
\hline 17 & & .51 & & & \\
\hline 18 & & .68 & & & \\
\hline 19 & & & & .87 & \\
\hline 21 & & & .75 & & \\
\hline 22 & & .60 & & & \\
\hline 23 & .69 & & & & \\
\hline 24 & & & .69 & & \\
\hline 25 & & .69 & & & \\
\hline 26 & & & & & .75 \\
\hline 27 & .33 & & & & \\
\hline 28 & & & & .42 & \\
\hline 29 & & & & & .61 \\
\hline 30 & & & .61 & & \\
\hline 31 & & & & & .45 \\
\hline
\end{tabular}

\begin{tabular}{llllll}
\hline $\begin{array}{l}\text { Desapego } \\
\text { Afecti- } \\
\text { vidad } \\
\text { Negativa }\end{array}$ & .54 & & & & \\
$\begin{array}{l}\text { Psicoticis- } \\
\text { mo }\end{array}$ & .52 & .68 & & & \\
$\begin{array}{l}\text { Antago- } \\
\text { nismo }\end{array}$ & .30 & .39 & .36 & & \\
$\begin{array}{l}\text { Desinhi- } \\
\text { bición }\end{array}$ & .49 & .68 & .63 & .52 & \\
$\boldsymbol{P}$ & .63 & .85 & .83 & .48 & .78 \\
\hline
\end{tabular}






Figura 2

Modelo bifactor para la versión al castellano del PID-5.

FE tiene una carga de .10 pero en el FG posee una de .64; o del ítem 27 que en el FE tiene una carga de .55 y en el FG una de .11). En general, las cargas factoriales en los FEs son más bajas que en los Modelos 1 y 2, pero esto es esperable ya que una vez que se divide la varianza para el factor general, queda menos varianza común. Los FEs representan covarianzas entre los elementos después de controlar el FG. 
Respecto a los índices estadísticos del modelo bifactor, el valor de coeficiente $\mathrm{H}$ del FG fue satisfactorio: .90. En cuanto al H de cada FE, desapego y antagonismo alcanzan valores satisfactorios, y el resto más bajos (desapego $=.70$; antagonismo $=.87 ;$ psicoticismo $=.63 ;$ afectividad negativa $=.60$; desinhibición $=.53)$. El $\omega \mathrm{h}$ del FG fue aceptable: .78, pero, tal como se esperaba, no llega al punto de corte de .80 que permitiría considerar al PID-5 como unidimensional. En cuanto a las $\omega \mathrm{hs}$, alcanzan valores satisfactorios, a excepción de afectividad negativa y psicoticismo $($ desapego $=.53 ;$ antagonismo $=.63 ;$ psicoticis$m o=.29 ;$ afectividad negativa $=.26$; desinhibición $=.31)$. Si bien para autores como Smits et al. (2014) serían aceptables (entre .30 y .20 refleja proporción moderada de varianza), para Arias et al. (2018) valores por debajo de .50 impiden la interpretación del factor. Por último, si bien el PUC es alto (.82), la magnitud del ECV fue inferior a $.70(.50)$, lo cual sugiere nuevamente que los datos no son lo suficientemente unidimensionales (Rodriguez et al., 2016). En cuanto al ECV-I, siete ítems (ver Tabla 5), tendrían una influencia significativa del FG: 8, 18 y 25 (afectividad negativa), 4 y 11 (psicoticismo), 29 (desinhibición) y 3 (desapego). Se trata de ítems que son explicados esencialmente por el FG y son mejores indicadores del FG que de su factor específico. Por otro lado, hay algunos ítems que parecen ser buenos indicadores tanto del FG como del FE, con valores correspondientes de ECV-I alrededor de .50 (ítems 1, 7, 14, 16, y 30; ver Rodriguez et al., 2016).

Finalmente el Modelo 4, unidimensional, no presentó buen ajuste a los datos: $\chi^{2}=4039.20_{(405)}$, $p<.001 ; \mathrm{S}-\mathrm{B} \chi^{2}=2061.79 ; \mathrm{CFI}=.85$; $\mathrm{RMSEA}=$ $.102,90 \% \mathrm{IC}=[.098 ; .107] ; \mathrm{GFI}=.876 ; \mathrm{AGFI}=$ .857 ; IFI $=.850 ; \mathrm{NFI}=.820 ; \mathrm{NNFI}=.839$. Esto indica que un único valor no sería suficiente para explicar los resultados. Las cargas factoriales oscilaron entre .20 y .69 (Tabla 6).
Tabla 5

Cargas factoriales para el Modelo 3, bifactor.

\begin{tabular}{|c|c|c|c|c|c|c|c|}
\hline & $\begin{array}{c}\text { Desape- } \\
\text { go }\end{array}$ & $\begin{array}{c}\text { Afecti- } \\
\text { vidad } \\
\text { negativa }\end{array}$ & $\begin{array}{c}\text { Psicoti- } \\
\text { cismo }\end{array}$ & $\begin{array}{c}\text { Antago- } \\
\text { nismo }\end{array}$ & $\begin{array}{c}\text { Desinhi- } \\
\text { bición }\end{array}$ & $\begin{array}{c}\text { Factor } \\
\text { General } \\
(p)\end{array}$ & ECV-I \\
\hline 1 & & & & & .47 & .46 & .49 \\
\hline 2 & & .67 & & & & .25 & .12 \\
\hline 3 & .30 & & & & & .59 & .80 \\
\hline 4 & & & .16 & & & .71 & .95 \\
\hline 5 & .54 & & & & & .44 & .39 \\
\hline 6 & & & & .42 & & .30 & .33 \\
\hline 7 & .45 & & & & & .44 & .49 \\
\hline 8 & & .12 & & & & .46 & .94 \\
\hline 9 & & & & .68 & & .47 & .32 \\
\hline 10 & & & & & .55 & .49 & .44 \\
\hline 11 & & & .24 & & & .59 & .86 \\
\hline 12 & & .35 & & & & .23 & .30 \\
\hline 13 & .61 & & & & & .23 & .12 \\
\hline 14 & & .44 & & & & .44 & .50 \\
\hline 15 & & & & & .29 & .22 & .36 \\
\hline 16 & & & .57 & & & .51 & .44 \\
\hline 17 & & .30 & & & & .43 & .67 \\
\hline 18 & & .14 & & & & .62 & .95 \\
\hline 19 & & & & .92 & & .39 & .15 \\
\hline 21 & & & .40 & & & .63 & .71 \\
\hline 22 & & .38 & & & & .50 & .63 \\
\hline 23 & .60 & & & & & .42 & .32 \\
\hline 24 & & & .63 & & & .51 & .39 \\
\hline 25 & & .10 & & & & .64 & .98 \\
\hline 26 & & & & & .42 & .59 & .66 \\
\hline 27 & .55 & & & & & .11 & .03 \\
\hline 28 & & & & .44 & & .16 & .11 \\
\hline 29 & & & & & .19 & .51 & .94 \\
\hline 30 & & & .46 & & & .47 & .51 \\
\hline 31 & & & & & .27 & .35 & .62 \\
\hline
\end{tabular}


Tabla 6

Cargas factoriales en el Modelo 4, unidimensional.

\begin{tabular}{ccccc}
\hline Item & Factor & & Item & Factor \\
\cline { 5 - 5 } 1 & .50 & & 16 & .59 \\
2 & .32 & & 17 & .44 \\
3 & .57 & & 18 & .59 \\
4 & .69 & & 19 & .59 \\
5 & .50 & & 21 & .66 \\
6 & .35 & & 22 & .52 \\
7 & .48 & & 23 & .49 \\
8 & .43 & & 24 & .60 \\
9 & .64 & & 25 & .59 \\
10 & .53 & & 26 & .59 \\
11 & .58 & & 27 & .20 \\
12 & .25 & & 28 & .23 \\
13 & .32 & 29 & .50 \\
14 & .46 & 30 & .52 \\
15 & .25 & & 31 & .36 \\
\hline
\end{tabular}

\section{Discusión}

La psicopatología tradicional, que iba de los conceptos clasificatorios a los datos, se encuentra en una crisis sin retorno. Distintos modelos dimensionales, que van de los datos a los conceptos, se están evaluando como sus posibles reemplazantes. En esa línea, aparece también el modelo dimensional para los trastornos de personalidad de la APA, variante psicopatológica del modelo de los "cinco grandes". Sin embargo, aún no ha surgido un modelo hegemónico que subsuma a los distintos esfuerzos, tal como lo hicieron los cinco grandes factores en los años 80 respecto a la personalidad (Sanchez \& Ledesma, 2007). Este trabajo se inserta dentro de esa metodología, y busca aportar evidencia sobre la dimensionalidad de rasgos patológicos en nuestra cultura, en población general. Para ello, se analiza la estructura subyacente a esos rasgos y se comparan diferentes modelos alternativos mediante AFC. A par- tir de los datos obtenidos con una versión local del PID-5, instrumento diseñado para evaluar las dimensiones de personalidad patológica, el modelo bifactor fue el que mejor representó la naturaleza de los rasgos subyacentes. Este modelo tuvo mejor ajuste tanto respecto al modelo original de cinco factores correlacionados como al de segundo orden (con un factor de orden superior). Por su parte, el modelo unidimensional fue el de peor ajuste a los datos, por lo que en sí mismo no resulta suficiente para describir la estructura subyacente. Los índices complementarios al modelo bifactor (p. ej., ECV, wh) van también en esa misma dirección, lo que indica que la estructura del PID no se acerca a una medida unidimensional.

Los resultados en conjunto sugieren que tanto las cinco dimensiones como un factor general $p$ aportan información de relevancia para la comprensión de los datos. Además de los cinco rasgos de personalidad patológicos (que corresponden al modelo teórico alternativo del DSM-5) habría un factor general, que reflejaría una propensión general a la psicopatología. El hecho de que en casi todos los factores (a excepción de antagonismo) haya uno o dos ítems que son explicados esencialmente por el FG (ECV-I alto), podría ser consistente con la idea de Caspi y Moffitt (2018) de que el FG representa un factor de riesgo común para desarrollar todas y cada una de las formas de psicopatología.

Los hallazgos de este estudio además se corresponden con las propuestas jerárquicas y dimensionales de la psicopatología (Conway et al., 2019; Kotov et al., 2017; Krueger et al., 2018). De esta manera, pueden contribuir a una mejor comprensión de la estructura subyacente a los trastornos del modelo dimensional de la APA, evaluados con el PID-5, sea en su versión original (Krueger et al., 2012) como en la versión castellana (Sanchez et al., en prensa). 
Limitaciones y líneas de investigación futuras

Los resultados, si bien son satisfactorios, se deben interpretar con cautela debido a ciertas limitaciones intrínsecas del estudio. En primer lugar, estos hallazgos se basan en una versión preliminar del instrumento que requiere mayores evidencias de validez. El hecho de tratarse de una versión breve del instrumento original puede restarle alcance a las conclusiones que puedan derivarse del presente trabajo. En este sentido, los resultados habilitan a continuar con el estudio, pero no permiten arribar a conclusiones sólidas respecto a la validez del modelo. Por el contrario, resulta necesario volver a administrar el instrumento, incluyendo además nuevos ítems para cubrir las diferentes facetas del modelo de la APA (2013). Del mismo modo, también habría que volver a probar ciertos ítems que no han tenido buenas propiedades psicométricas y que no se han considerado en esta muestra.

El propio modelo bifactor también presenta algunos inconvenientes que deben tenerse en cuenta. Como se expresó anteriormente, un buen resultado estadístico no garantiza la existencia de un factor general (Bonifay et al., 2017). Por otro lado, si bien el bifactor es el que posee el mejor ajuste, el modelo de segundo orden (M2) es levemente más parsimonioso, lo que se corresponde con la evidencia que sostiene que estadísticamente ambos modelos son satisfactorios (Ringwald et al., 2019).

La elección y el tamaño de la muestra también suponen un límite a los alcances de este trabajo. Dado que se trata de un instrumento que evalúa psicopatología, resulta necesaria su administración en otras muestras, en particular clínicas. Aquí se trabajó con una muestra de población general, en función del objetivo de contar con una primera aproximación al funcionamiento del instrumento. Sin embargo, en tal población posiblemente no han de encontrarse rasgos psicopatológicos al mismo nivel que en una población clínica; por tanto, no debe considerarse, en principio, que la estructura psicopatológica se exprese de la misma forma en ambas poblaciones. Además, al no haberse seguido criterios estrictos en la elección de los participantes, la muestra resultante muestra una gran heterogeneidad. Tales cuestiones se deberían dirimir en futuros trabajos con muestras diversas e incluso con un mayor número de participantes.

El propio factor $p$ requiere una interpretación más profunda. Manteniendo el símil con el factor $g$ de inteligencia, todos tendríamos un valor de $p$ de bajo a alto que indicaría nuestra propensión a padecer trastornos psicológicos de mayor o menor gravedad, y de mayor o menor duración. Por tanto, todos presentaríamos un valor de $p$ que, cuanto más elevado, más propensos nos haría a sufrir trastornos graves, crónicos o recurrentes.

Por último, si bien los hallazgos son consonantes con la propuesta de Caspi et al. (2014) y Caspi y Moffitt (2018) y con el HiTOP (Kotov et al., 2017), se trata de modelos recientes que también necesitan mayor validación. La psicopatología es un campo complejo y se encuentra en medio de una crisis kuhniana. En este panorama, las herramientas psicométricas intentan echar un poco de luz sobre el campo, lo que ayuda a formular posibles modelos psicopatológicos más válidos que el tradicional de categorías. El objetivo final sigue siendo contar con un modelo psicopatológico que dé cuenta de forma más acabada del malestar psicológico y que preste utilidad clínica. $\mathrm{Si}$ bien el andamiaje estadístico colabora en ese cometido, no debe perderse de vista la necesidad de sustentar teóricamente cualquier hallazgo. Solo estudios posteriores podrán determinar qué propuesta resulta más comprehensiva, teórica y psicométricamente, para reemplazar las agotadas categorías de la nomenclatura oficial (APA, 2013). 
En síntesis, a pesar de las limitaciones señaladas, los resultados muestran que las propuestas jerárquicas y dimensionales, que postulan un factor general junto a factores específicos, abren un campo prometedor para el estudio de la psicopatología, por lo que puede sostenerse que, si bien la crisis continúa, el reemplazo del modelo categorial está en camino.

\section{Referencias}

Al-Dajani, N., Gralnick, T. M., \& Bagby, R. M. (2016). A psychometric review of the Personality Inventory for DSM-5 (PID-5): Current status and future directions. Journal of Personality Assessment, 98(1), 62-81. doi: 10.1080/00223891.2015.1107572

American Psychiatric Association. (1952). Diagnostic and statistical manual of mental disorders. Washington, D. C.: Author.

American Psychiatric Association. (1980). Diagnostic and statistical manual of mental disorders. DSM-III ( $3^{\mathrm{a}}$ ed.). Washington, D. C.: Author.

American Psychiatric Association. (1994). Diagnostic and statistical manual of mental disorders. DSM-IV ( $4^{\mathrm{a}}$ ed.). Washington, D. C.: Author.

American Psychiatric Association. (2013). Diagnostic and statistical manual of mental disorders. DSM-5 ( $5^{\mathrm{a}}$ ed.). Washington, D. C.: Author. doi: 10.1176/appi. books.9780890425596

Arias, V. B., Ponce, F. P., \& Núñez, D. E. (2018). Bifactor models of attention-deficit/hyperactivity disorder (ADHD): An evaluation of three necessary but underused psychometric indexes. Assessment, 25(7), 885-897. doi: 10.1177/1073191116679260

Batstra, L., \& Thoutenhoofd, E. D. (2012). The risk that DSM-5 will further inflate the diagnostic bubble. Current Psychiatry Reviews, 8(4), 260-263. doi: 10.2174/157340012803520531

Blashfield, R., Flanagan, E., \& Raley, K. (2010). Themes in the evolution of the 20th-Century DSMs. En
T. Millon, R. F. Krueger \& E. Simonsen (Eds.), Contemporary directions in psychopathology: Scientific foundations of the DSM-V and ICD-11 (pp. 53-71). New York, NY: Guilford.

Bonifay, W., Lane, S. P., \& Reise, S. P. (2017). Three concerns with applying a bifactor model as a structure of psychopathology. Clinical Psychological Science, 5(1), 184-186. doi: 10.1177/2167702616657069

Browne, M. W., \& Cudeck, R. (1992). Alternative ways of assessing model fit. Sociological Methods \& Research, 21(2), 230-258. doi: 10.1177/0049124192021002005

Caspi, A., Houts, R. M., Belsky, D. W., Goldman-Mellor, S. J., Harrington, H. L., Israel, S., ... Moffitt, T. E. (2014). The $p$ Factor: One general psychopathology factor in the structure of psychiatric disorders? Clinical Psychological Science, 2(2), 119-137. doi: $10.1177 / 2167702613497473$

Caspi, A., \& Moffitt, T. E. (2018). All for one and one for all: Mental disorders in one dimension. The American Journal of Psychiatry, 175(9), 831-844. doi: 10.1176/appi.ajp.2018.17121383

Conway, C. C., Forbes, M. K., Forbush, K. T., Fried, E. I., Hallquist, M. N., Kotov, R., ... Eaton, N. R. (2019). A hierarchical taxonomy of psychopathology can transform mental health research. Perspectives on Psychological Science, 14(3), 419-436. doi: 10.1177/1745691618810696

Coolidge, F. L., \& Segal, D. L. (1998). Evolution of personality disorder diagnosis in the Diagnostic and Statistical Manual of Mental Disorders. Clinical Psychology Review, 18(5), 585-599. doi: 10.1016/ S0272-7358(98)00002-6

Costa, P. T., \& Widiger, T. A. (1994). Personality disorders and the five-factor model of personality. Washington, DC: American Psychological Association. Recuperado de doi: 10.1037/10140-000

Dominguez-Lara, S. A. (2016). Evaluación de la confiabilidad del constructo mediante el coeficiente $\mathrm{H}$ : Breve revisión conceptual y aplicaciones. Psychologia. Avances de la Disciplina, 10(2), 87-94. doi: $10.21500 / 19002386.2134$ 
Dominguez-Lara, S. A., \& Rodriguez, A. (2017). Índices estadísticos de modelos bifactor. Interacciones, 3, 59-65. doi: 10.24016/2017.v3n2.51

Flores-Kanter, P. E., Dominguez-Lara, S., Trógolo, M. A., \& Medrano, L. A. (2018). Best practices in the use of bifactor models: Conceptual grounds, fit indices and complementary indicators. Revista Evaluar, 18(3), 44-48. doi: 10.35670/1667-4545.v18.n3.22221

Forbes, M. K., Baillie, A. J., \& Schniering, C. A. (2015). Should sexual problems be included in the internalising spectrum? A comparison of dimensional and categorical models. Journal of Sex \& Marital Therapy, 42(1), 70-90. doi: 10.1080/0092623X.2014.996928

Frances, A. (2010). Opening Pandora's box: The 19 worst suggestions for DSM5. Psychiatric Times, 27(9).

Frances, A. (2014). ¿Somos todos enfermos mentales? Manifiesto contra los abusos de la Psiquiatría. Barcelona, España: Ariel.

Gagne, P., \& Hancock, G. R. (2006). Measurement model quality, sample size, and solution propriety in confirmatory factor models. Multivariate Behavioral Research, 41(1), 65-83. doi: 10.1207/ s15327906mbr4101_5

Gutiérrez, F., Aluja, A., Peri, J. M., Calvo, N., Ferrer, M., Baillés, E., ... Krueger, R. F. (2017). Psychometric properties of the Spanish PID-5 in a clinical and a community sample. Assessment, 24(3), 326-336. doi: 10.1177/1073191115606518

Hancock, G. R. (2001). Effect size, power, and sample size determination for structured means modeling and MIMIC approaches to between-groups hypothesis testing of means on a single latent construct. Psychometrika, 66(3), 373-388. doi: 10.1007/ BF02294440

Haslam, N., Holland, E., \& Kuppens P. (2012). Categories versus dimensions in personality and psychopathology: A quantitative review of taxometric research. Psychological Medicine, 42(5), 903-920. doi: 10.1017/S0033291711001966

Holzinger, K. J., \& Swineford, F. (1937). The bi-factor method. Psychometrika, 2(1), 41-54. doi: 10.1007/ bf02287965

Hopwood, C. J., Malone, J. C., Ansell, E. B., Sanislow, C. A., Grilo, C. M., McGlashan, T. H., ... Morey, L. C. (2011). Personality assessment in DSM-V: Empirical support for rating severity, style, and traits. Journal of Personality Disorders, 25(3), 305-320. doi: 10.1521/ pedi.2011.25.3.305

Hoyle, R. H.. (2012). Handbook of structural equation modeling. New York, NY: Guilford Press.

Hu, L., \& Bentler, P. M. (1999). Cutoff criteria for fit indexes in covariance structure analysis: Conventional criteria versus new alternatives. Structural Equation Modeling: A Multidisciplinary Journal, 6(1), 1-55. doi: 10.1080/10705519909540118

John, O. P., \& Srivastava, S. (1999). The Big Five Trait taxonomy: History, measurement, and theoretical perspectives. En L. A. Pervin \& O. P. John (Eds.), Handbook of personality: Theory and research (pp. 102-138). New York, NY: Guilford.

Jöreskog, K. G., \& Sörbom, D. (2006). LISREL 8.80. Chicago: Scientific Software International.

Kessler, R. C., Chiu, W. T., Demler, O., \& Walters, E. E. (2005). Prevalence, severity, and comorbidity of 12-month DSM-IV disorders in the National Comorbidity Survey Replication. Archives of General Psychiatry, 62(6), 617-627. doi: 10.1001/ archpsyc.62.6.617

Kotov, R., Krueger, R. F., Watson, D., Achenbach, T. M., Althoff, R. R., Bagby, R. M., ... Zimmerman, M. (2017). The Hierarchical Taxonomy of Psychopathology (HiTOP): A dimensional alternative to traditional nosologies. Journal of Abnormal Psychology, 126(4), 454-477. doi: 10.1037/ abn0000258

Krueger, R. F., Derringer, J., Markon, K. E., Watson, D., \& Skodol, A. E. (2012). Initial construction of a maladaptive personality trait model and inventory for DSM-5. Psychological Medicine, 42(9), 1879-1890. doi: 10.1017/S0033291711002674

Krueger, R. F., Kotov, R., Watson, D., Forbes, M. K., Eaton, N. R., Ruggero, C. J., ... Zimmermann, J. (2018). 
Progress in achieving quantitative classification of psychopathology. World Psychiatry, 17(3), 282-293. doi: 10.1002/wps.20566

Kupfer, D. J., First, M. B., \& Regier, D. A. (Eds.). (2002). A research agenda for $D S M-V$. Washington, D. C.: American Psychological Association.

Lahey, B. B., Applegate, B., Hakes, J. K., Zald, D. H., Hariri, A. R., \& Rathouz, P. J. (2012). Is there a general factor of prevalent psychopathology during adulthood? Journal of Abnormal Psychology, 121, 971-977. doi: 10.1037/a0028355

Lahey, B. B., Krueger, R. F., Rathouz, P. J., Waldman, I. D., \& Zald, D. H. (2017). A hierarchical causal taxonomy of psychopathology across the life span. Psychological Bulletin, 143(2), 142-186. doi: 10.1037/bul0000069

Li, C. H. (2016). Confirmatory factor analysis with ordinal data: Comparing robust maximum likelihood and diagonally weighted least squares. Behavior Research Methods, 48(3), 936-949. doi: 10.3758/ s13428-015-0619-7

Livesley, J. (2012). Tradition versus empiricism in the current DSM-5 proposal for revising the classification of personality disorders. Criminal Behaviour and Mental Health, 22(2), 81-90. doi: 10.1002/cbm.1826

Lorenzo-Seva, U., \& Ferrando, P. J. (2013). FACTOR 9.2: A comprehensive program for fitting exploratory and semiconfirmatory factor analysis and IRT models. Applied Psychological Measurement, 37(6), 497498. doi: $10.1177 / 0146621613487794$

Mayes, R., \& Horwitz, A. V. (2005). DSM-III and the revolution in the classification of mental illness. Journal of the History of the Behavioral Sciences, 41(3), 249267. doi: 10.1002/jhbs.20103

Mardia, K. V. (1970). Measures of multivariate skewness and kurtosis with applications. Biometrika, 57(3), 519-530. doi: $10.2307 / 2334770$

Millon, T. (1983). The DSM-III: An insider's perspective. American Psychologist, 38(7), 804-814. doi: 10.1037/0003-066X.38.7.804

Mîndrilă, D. (2010). Maximum Likelihood (ML) and
Diagonally Weighted Least Squares (DWLS) estimation procedures: A comparison of estimation bias with ordinal and multivariate non-normal data. International Journal of Digital Society, 1(1), 60-66. doi: 10.20533/ijds.2040.2570.2010.0010

Muthukrishna, M., \& Henrich, J. (2019). A problem in theory. Nature Human Behaviour, 3(3), 221-229. doi: 10.1038/s41562-018-0522-1

Paris, J. (2012). The risk that DSM-5 will give personality dimensions a bad name. Current Psychiatry Reviews, 8(4), 268-270. doi: 10.2174/157340012803520441

Patrick, C. J., Hicks, B. M., Nichol, P. E., \& Krueger, R. F. (2007). A bifactor approach to modeling the structure of the Psychopathy Checklist-Revised. Journal of Personality Disorders, 21(2), 118-141. doi: 10.1521/ pedi.2007.21.2.118

Quinn, H. O. (2014). Bifactor models, Explained Common Variance (ECV), and the usefulness of scores from unidimensional item response theory analyses. (Tesis de Maestría Inédita). University of North Carolina at Chapel Hill, Chapel Hill, NC.

Reise, S. P. (2012). The rediscovery of bifactor measurement models. Multivariate Behavioral Research, 47(5), 667-696. doi: 10.1080/00273171.2012.715555

Reise, S. P., Kim, D. S., Mansolf, M., \& Widaman, K. F. (2016). Is the bifactor model a better model or is it just better at modeling implausible responses? Application of iteratively reweighted least squares to the Rosenberg Self-Esteem scale. Multivariate Behavioral Research, 51(6), 818-838. doi: 10.1080/00273171.2016.1243461

Ringwald, W. R., Beeney, J. E., Pilkonis, P. A., \& Wright, A. G. (2019). Comparing hierarchical models of personality pathology. Journal of Research in Personality, 81, 98-107. doi: 10.1016/j.jrp.2019.05.011

Rodriguez, A., Reise, S. P., \& Haviland, M. G. (2016). Evaluating bifactor models: Calculating and interpreting statistical indices. Psychological Methods, 21(2), 137-150. doi: 10.1037/met0000045

Sanchez, R. O. (en prensa). Modelos dimensionales para los trastornos de la personalidad: Un proceso incon- 
cluso. Revista Argentina de Clínica Psicológica. doi: 10.24205/03276716.2019.1126

Sanchez, R. O., \& Ledesma, R. (2007). Los Cinco Grandes Factores: Cómo entender la personalidad y cómo evaluarla. En A. Monjeau (Ed.), Conocimiento para la transformación (pp. 131-160). Mar del Plata, Argentina: Universidad Atlántida Argentina.

Sánchez, R. O. \& Montes, S. A. (2019). La taxonomía jerárquica y el factor general de psicopatología. Acta Psiquiátrica y Psicológica de América Latina, 65(2), 116-129.

Sanchez, R. O., Montes, S. A., \& Somerstein, L. D. (en prensa). Inventario de Personalidad para el DSM-5: Propiedades psicométricas en población argentina. Estudio preliminar. Interdisciplinaria.

Satorra, A., \& Bentler, P. M. (1990). Model conditions for asymptotic robustness in the analysis of linear relations. Computational Statistics \& Data Analysis, 10(3), 235-249. doi: 10.1016/0167-9473(90)90004-2

Sharp, C., Wright, A. G. C., Fowler, J. C., Frueh, B. C., Allen, J. G., Oldham, J., \& Clark, L. A. (2015). The structure of personality pathology: Both general (' $g$ ') and specific (' $s$ ') factors? Journal of Abnormal Psychology, 124(2), 387-398. doi: 10.1037/ abn0000033

Shedler, J., Beck, A., Fonagy, P., Gabbard, G. O., Gunderson, J., Kernberg, O., ... Westen, D. (2010). Personality disorders in DSM-5. The American Journal of Psychiatry, 167(9), 1026-1028. doi: 10.1176/appi. ajp.2010.10050746

Sijtsma, K. (2009). On the use, the misuse, and the very limited usefulness of Cronbach's alpha. Psychometrika, 74(1), 107-120. doi: 10.1007/s11336-008-9101-0

Snyder, H. R., Young, J. F., \& Hankin, B. L. (2017). Strong homotypic continuity in common psychopathology-, internalizing-, and externalizing-specific factors over time in adolescents. Clinical Psychological Science, 5(1), 98-110. doi: 10.1177/2167702616651076

Smits, I. A. M., Timmerman, M. E., Barelds, D. P. H., \& Meijer, R. R. (2015). The Dutch symptom checklist-90-revised: Is the use of the subscales justified?
European Journal of Psychological Assessment, 31(4), 263-271. doi: 10.1027/1015-5759/a000233

Stucky, B. D., Thissen, D., \& Edelen, M. O. (2013). Using logistic approximations of marginal trace lines to develop short assessments. Applied Psychological Measurement, 37(1), 41-57. doi: $10.1177 / 0146621612462759$

Ten-Berge, J. M., \& Sočan, G. (2004). The greatest lower bound to the reliability of a test and the hypothesis of unidimensionality. Psychometrika, 69(4), 613-625. doi: 10.1007/BF02289858

Tyrer, P. (2005). The problem of severity in the classification of personality disorder. Journal of Personality Disorders, 19(3), 309-314. doi: 10.1521/ pedi.2005.19.3.309

Urbán, R., Arrindell, W. A., Demetrovics, Z., Unoka, Z., \& Timman, R. (2016). Cross-cultural confirmation of bi-factor models of a symptom distress measure: Symptom Checklist-90-Revised in clinical samples. Psychiatry Research, 239, 265-274. doi: 10.1016/j. psychres.2016.03.039

Van de Schoot, R., Lugtig, P., \& Hox, J. (2012). A checklist for testing measurement invariance. European Journal of Developmental Psychology, 9(4), 486492. doi: 10.1080/17405629.2012.686740

Verheul, R. (2012). Personality disorder proposal for DSM-5: A heroic and innovative but nevertheless fundamentally flawed attempt to improve DSM-IV. Clinical Psychology \& Psychotherapy, 19(5), 369371. doi: 10.1002/cpp.1809

Waszczuk, M. A., Kotov, R., Ruggero, C., Gamez, W., \& Watson, D. (2017). Hierarchical structure of emotional disorders: From individual symptoms to the spectrum. Journal of Abnormal Psychology, 126(5), 613-634. doi: 10.1037/abn0000264

Watters, C. A., \& Bagby, R. M. (2018). A meta-analysis of the five-factor internal structure of the Personality Inventory for DSM-5. Psychological Assessment, 30(9), 1255-1260. doi: 10.1037/pas0000605

Widiger, T. (2011). A shaky future for personality disorders. Personality Disorders: Theory, Research, and 
Treatment, 2(1), 54-67. doi: 10.1037/a0021855

Widiger, T. (2013). A postmortem and future look at the personality disorders in DSM-5. Personality Disorders: Theory, Research, and Treatment, 4(4), 382-387. doi: 10.1037/per0000030

Zinbarg, R. E., Yovel, I., Revelle, W., \& McDonald, R. P. (2006). Estimating generalizability to a latent variable common to all of a scale's indicators: A comparison of estimators for $\omega$ h. Applied Psychological Measurement, 30(2), 121-144. doi: 10.1177/0146621605278814 